\title{
COMPACT DIAGONAL LINEAR OPERATORS ON BANACH SPACES WITH UNCONDITIONAL BASES
}

\author{
YUN SUNG CHOI and SUNG GUEN KIM \\ Department of Mathematics \\ Pohang Institute of Science and Technology \\ Pohang, Korea \\ (Received April 13, 1992 and in revised form July 3, 1992)
}

\begin{abstract}
Let $E$ and $F$ be Banach spaces with equivalent normalized unconditional bases. In this note we show that a bounded diagonal linear operator $T: E \rightarrow F$ is compact if and only if its entries tend to 0 , using the concept of weak uniform continuity.
\end{abstract}

KEY WORDS and PHRASES. Weakly uniformly continuous, diagonal operators, compact operators, unconditional bases.

1992 AMS SUBJECT CLASSIFICATION CODES. 47B05, $47 \mathrm{~B} 37$.

1. Introduction.

Let $E$ and $F$ be two complex Banach spaces and $E^{*}$ be the dual space of $E$. A function $f: E \rightarrow F$ is said to be weakly uniformly continuous on bounded subsets of $E$ if for each bounded set $B \subset E$ and $\epsilon>0$, there are $\phi_{1}, \cdots, \phi_{k} \in E^{*}$ and $\delta>0$ such that if $x, y \in B,\left|\phi_{\imath}(x-y)\right| \leq$ $\delta(i=1, \cdots, k)$, then $\|f(x)-f(y)\| \leq \epsilon$. R.M. Aron and J.B. Prolla [1] showed that a bounded linear operator $T: E \rightarrow F$ is compact if and only if $T$ is weakly uniformly continuous on bounded subsets of $E$. Applying this result we generalize the following well-known Hilbert space fact to a Banach space with an unconditional basis: A diagonal bounded linear operator is compact if and only if its entries tend to 0. See, for example, [2, Proposition 4.6].

We recall some relevant definitions and results about a Banach space with an unconditional basis. Let $E$ be a complex Banach space with an unconditional basis $\left(e_{n}\right)$. For every choice of signs $\theta=\left(\theta_{n}\right)$, we have a bounded linear operator $M_{\theta}$ on $E$ defined by

$$
M_{\theta}\left(\sum a_{n} e_{n}\right)=\sum a_{n} \theta_{n} e_{n}
$$

The uniform bounded principle implies that the number $K=\sup \left\|M_{\theta}\right\|$ is finite, which is called the unconditional constant of $\left(e_{n}\right)$. Then for every choice of a complex sequence $\left(a_{n}\right)$ such that $\sum a_{n} e_{n}$ converges and every choice of a bounded complex sequence $\left(\alpha_{n}\right)$, we have

$$
\left\|\sum \alpha_{n} a_{n} e_{n}\right\| \leq 2 K\left(\sup \left|\alpha_{n}\right|\right)\left\|\sum a_{n} e_{n}\right\| \text {. }
$$

For details see [3]. 
2. Main Results.

THEOREM 1. Let $\left(\epsilon_{n}\right)$ and $\left(f_{n}\right)$ be equivalent normalized unconditional bases of $E$ and $F$, respectively. (iiven a bounded sequence $\left(\alpha_{n}\right)$, let $T: E \rightarrow F$ be the bounded linear operator with $T\left(\epsilon_{n}\right)=\alpha_{n} f_{n}$ for cach $n$. Then $T$ is compact if and only if $\alpha_{n} \rightarrow 0$.

Proof. Suppose $T$ is compact. Let $\left(P_{n}\right)$ be the sequence of the natural projections associated with $\left(f_{n}\right)$. Then $\left(P_{n} \circ T\right)$ converges uniformly to $T$ on the closed unit ball $B_{E}$, from which it follows that $\alpha_{n} \rightarrow 0$.

Conversely suppose that $\alpha_{n} \rightarrow 0$. We will show that $T$ is weakly uniformly continuous on bounded subsets of $E$. Let $B_{r}$ be the closed ball of $E$ with the radius $r$ and the center 0 and $C$ be the positive number with $\left|\alpha_{n}\right| \leq C$ for all $n$. Given $\epsilon>0, x=\sum a_{n} e_{n}$ and $y=\sum b_{n} e_{n}$ in $B_{r}$.

$$
\begin{aligned}
\|T(x)-T(y)\| & =\left\|\sum_{n} \alpha_{n}\left(a_{n}-b_{n}\right) f_{n}\right\| \\
& \leq C \sum_{n=1}^{N-1}\left|a_{n}-b_{n}\right|+\left\|\sum_{n=N}^{\infty} \alpha_{n}\left(a_{n}-b_{n}\right) f_{n}\right\| \\
& \leq C \sum_{n=1}^{N-1}\left|a_{n}-b_{n}\right|+2 K\left(\sup _{n \geq N}\left|\alpha_{n}\right|\right)\left\|\sum_{n=N}^{\infty}\left(a_{n}-b_{n}\right) f_{n}\right\|,
\end{aligned}
$$

where $K$ is the unconditional constant of $\left(f_{n}\right)$. Since $\left(e_{n}\right)$ and $\left(f_{n}\right)$ are equivalent, it is easy to see that

$$
\left\|\sum_{n=N}^{\infty}\left(a_{n}-b_{n}\right) f_{n}\right\| \leq 2(1+K) r\|T\| .
$$

Let $\left(f_{n}^{*}\right)$ be the sequence of coefficient functionals associated with $\left(f_{n}\right)$. Since $\alpha_{n} \rightarrow 0$, choosing sufficiently large $N$, we conclude that

$$
\|T(x)-T(y)\| \leq \epsilon
$$

if $\left|f_{1}^{*}(x-y)\right|, \cdots,\left|f_{N-1}^{*}(x-y)\right|$ are sufficiently small. Hence $T$ is weakly uniformly continuous on bounded subset of $E$.

From the above proof it is easy to see that the Banach space $c_{0}$ of null complex sequences is isomorphic with the Banach space of compact diagonal linear operators $T: E \rightarrow F$, where $E$ and $F$ are Banach spaces with equivalent unconditional bases. We would like to remark that if $\left(\epsilon_{n}\right)$ and $\left(f_{n}\right)$ are not equivalent, then given a bounded complex sequence $\left(\alpha_{n}\right)$, the map $T\left(e_{n}\right)=\alpha_{n} f_{n}$ is not necessarily extended to a bounded linear operator from $E$ into $F$. For example take $E=\ell_{2}, F=\ell_{1}$ and $\alpha_{n}=1$ for all $n$ with respect to the canonical bases of them.

ACKNOWLEDGEMENT. Both authors would like to thank the referees for helpful suggestions and remarks.

\section{REFERENCE}

1. ARON, R.M. and PROLLA, J.B. Polynomial approximation of differentiable functions on Banach spaces, J. Reine Angew. Math. 313 (1980), 195-216.

2. CONWAY, J.B. A Course in Functional Analysis, Springer-Verlag, New York-Berlin-HeidelbergTokyo, 1985.

3. LINDENSTRAUSS, J. and TZAFRIRI, L. Classical Banach Spaces I, Springer-Verlag, BerlinHeidelberg-New York, 1977. 


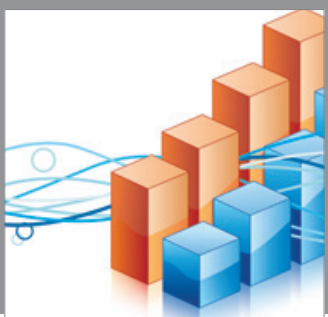

Advances in

Operations Research

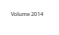

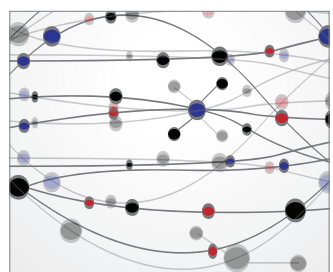

\section{The Scientific} World Journal
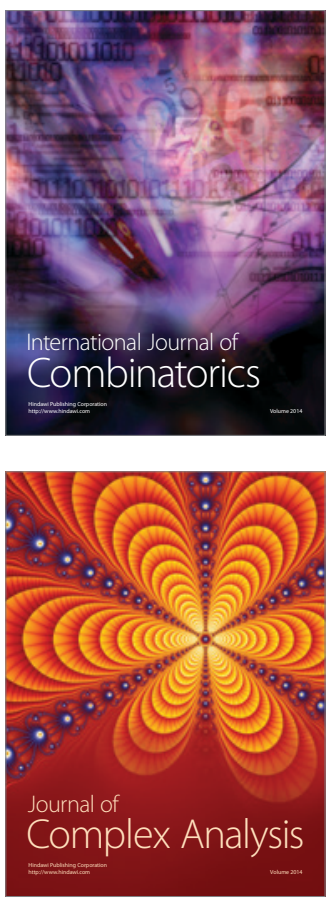

International Journal of

Mathematics and

Mathematical

Sciences
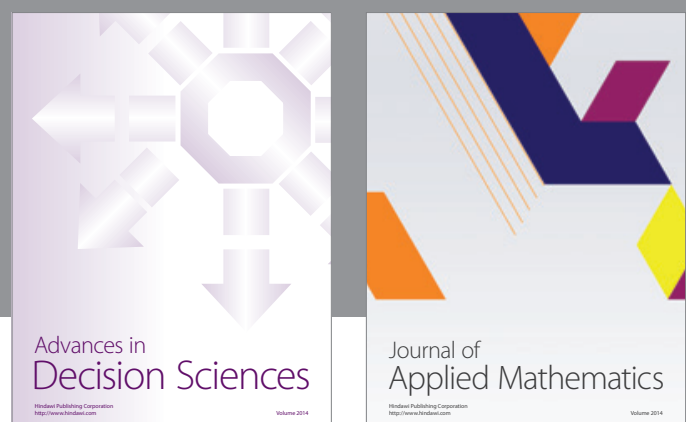

Journal of

Applied Mathematics
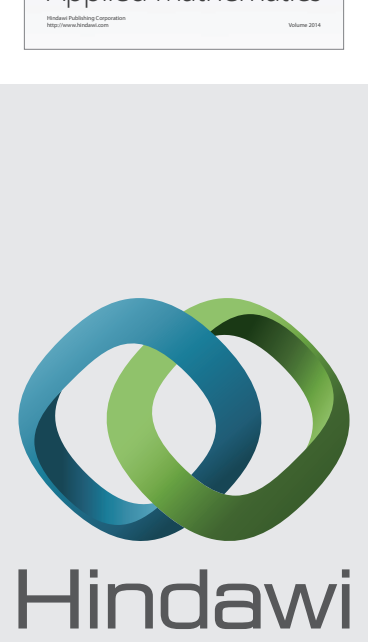

Submit your manuscripts at http://www.hindawi.com
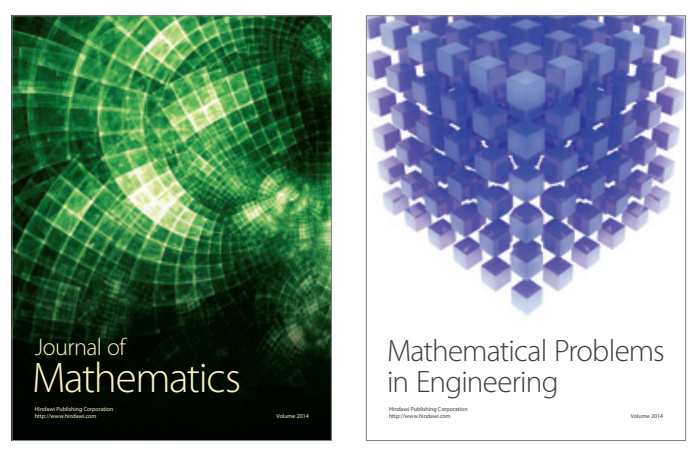

Mathematical Problems in Engineering
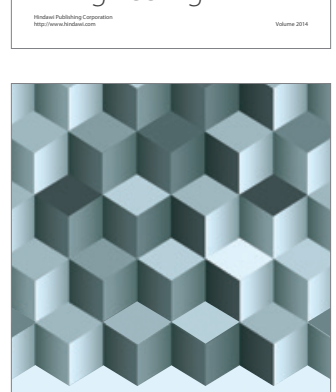

Journal of

Function Spaces
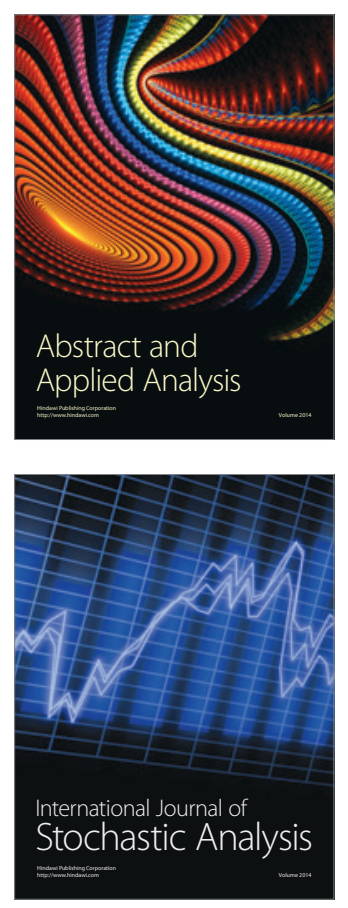

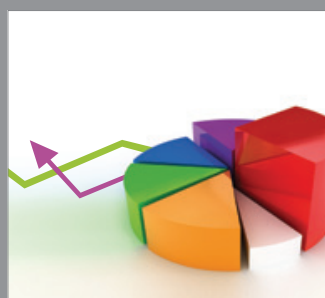

ournal of

Probability and Statistics

Promensencen
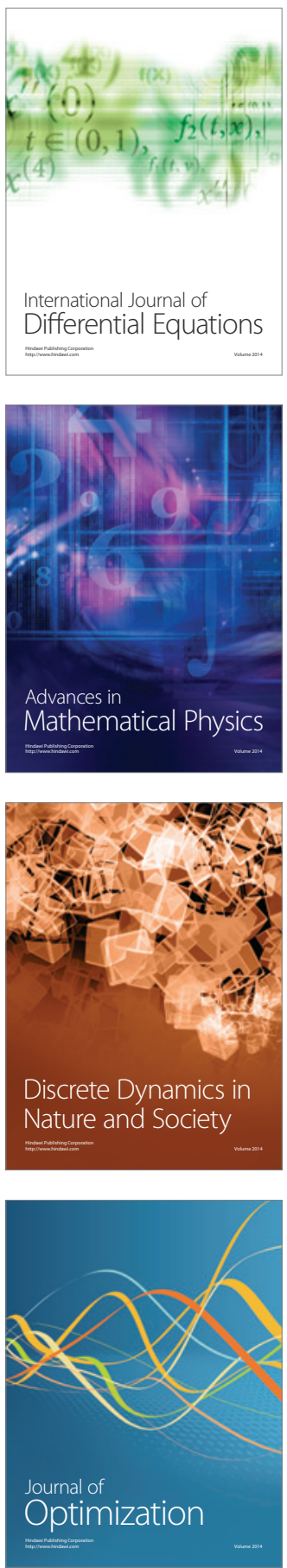